\title{
Vector Coherent State Realization of Representations of the Affine Lie Algebra $\widehat{s l}(2)$
}

\author{
R. B. ZHANG \\ Department of Pure Mathematics, University of Adelaide, Adelaide, Australia
}

\begin{abstract}
The method of vector coherent states is generalized to study representations of the affine Lie algebra $\widehat{s l}(2)$. A large class of highest weight irreps is explicitly constructed, which contains the integrable highest weight irreps as special cases.
\end{abstract}

\section{INTRODUCTION}

The method of vector coherent states was independently developed by the research groups of Quesne and Rowe[1] [2] to study representations of Lie groups appearing in physics. Applied to the irreducible representations of the compact semi - simple Lie groups, the method enables one to obtain explicit realizations of the generators of the corresponding Lie algebras in terms of holomorphic differential operators, and more importantly, the K - matrix technique [2] of the method allows the identification of the subsets of holomorphic polynomials which form the irreducible representation spaces. The method also provides a powerful machinery for constructing unitary representations of noncompact Lie groups; we refer to [2] for details on this subject. The method has also been extended to Lie superalgebras [0], and in recent years, to quantum groups and quantum supergroups 四].

A notable feature of the method of vector coherent states is that it is particularly well adapted to standard techniques in physics, thus is readily applicable to addressing concrete physical problems. Apart from its physical applications, the method is also of great mathematical interests. In particular, it is closely related [5] to the Bott - Borel - Weil theorem. The theorem is one of the hall marks in the representation theory of Lie groups. It realizes the finite dimensional irreps of compact Lie groups in terms of cohomology groups of homogeneous vector bundles. The Langlands and Kostant conjectures, proved by Schmid, generalize the theorem to noncompact semi - simple Lie groups, yielding a geometrical realization of Harish - Chandra's discrete series of representations. The Bott - Borel - Weil theory was further modified and significantly extended in the last 20 years, leading to the development of the theory of cohomological induction, which has now become a fundamental part of modern representation theory. The Bott - Borel - Weil theory also plays important roles in various other fields, most notably, geometric quantization and Penrose transforms. 
The aim of the present note is to develop the vector coherent state construction of representations for affine Lie algebras. We will consider affine $s l(2)$ only in this note; more general cases will be treated else where. Affine Lie algebras [6] made their first appearance in physics in the context of dual resonance models, now called strings ( See [7] for references on applications of affine Lie algebras to string theory.). Later studies showed that these algebras are also of paramount importance for conformal field theory and for two dimensional integrable models in statistical mechanics(See [8] for references.). In fact the investigation of their applications in these three areas has been a major theme of mathematical physics since the early 1980s. We should also mention that Wen proposed to utilize affine Lie algebras to describe the so - called edge states in fractional quantum Hall effect. We refer to Section 3 of Chapter 10 in Fradkin's book [9] for a concise discussion of Wen's proposal.

We generalize the vector coherent state method to explicitly construct a large class of highest weight irreps, which include the integrable highest weight irreps as special cases. The main results are summarized in equations (6) and (7), which we believe to be new. Our construction may be regarded as an infinitesimal version of the construction of Pressley and Segal's [10], which yields integrable highest weight irreps of loop groups as holomorphic sections of vector bundles over 'fundamental homogeneous spaces'. Our construction of $\widehat{s l}(2)$ irreps also bears considerable similarity with the widely studied Wakimoto construction (See, e.g., [11].), although it also differs from the latter in many essential ways. It will be interesting to find out what the exact relationship is between the two. Results presented here should extend to general affine Lie algebras and the Virasoro algebra, as well as their supersymmetric counter parts. We shall return to these problems in the future.

\section{AFFINE $s l(2)$ AND ITS REPRESENTATIONS}

We will work on the complex number field $\mathbb{C}$. The affine Lie algebra $\widehat{s l}(2)$ has a basis $\{\kappa ; e[n], h[n], f[n], n \in \mathbb{Z}\}$ satisfying the following commutation relations

$$
\begin{aligned}
{[h[m], e[n]] } & =2 e[m+n], \\
{[h[m], f[n]] } & =-2 f[m+n], \\
{[h[m], h[n]] } & =2 m \kappa \delta_{m+n, 0}, \\
{[e[m], f[n]] } & =h[m+n]+m \kappa \delta_{m+n, 0},
\end{aligned}
$$

the rest vanishes.

We will denote by $U(\widehat{s l}(2))$ its universal enveloping algebra, and regard $\widehat{s l}(2)$ as embedded in $U(\widehat{s l}(2))$. Note that this affine Lie algebra contains a number of interesting subalgebras, which will be useful for studying the representation theory. In particular, we have

$$
\begin{aligned}
s l(2) & =\langle e[0], h[0], f[0]\rangle, \\
\mathfrak{b}_{+} & =\langle\kappa ; e[0], h[0] ; e[n], h[n], f[n], n>0\rangle, \\
\mathfrak{p} & =\left\langle\kappa ; ; \quad e[n], h[n], f[n], n \in \mathbb{Z}_{+}\right\rangle, \\
\mathfrak{u}_{+} & =\langle e[n], h[n], f[n], n>0\rangle, \\
\mathfrak{u}_{-} & =\langle e[n], h[n], f[n], n<0\rangle,
\end{aligned}
$$


where $\mathfrak{b}_{+}$is a familiar Borel subalgebra, and $\mathfrak{p}$, containing $\mathfrak{b}_{+}$, will be called a parabolic subalgebra. Note also that $\widehat{s l}(2)=\mathfrak{u}_{-}+\mathfrak{p}$.

A standard technique in representation theory is to induce $\widehat{s l}(2)$ modules, i.e., Verma modules, from given one dimensional $\mathfrak{b}_{+}$modules. More generally, one can also start with finite dimensional highest weight $\mathfrak{p}$ modules to induce generalized Verma modules, and to obtain irreps as subquotients. This method is the analogue of the parabolic induction widely used in the representation theory of finite dimensional Lie algebras. It also provides the basis for developing the method of vector coherent states.

Let $V_{0}$ be a finite dimensional irreducible $\mathfrak{p}$ module such that the positive modes $e[n], h[n], f[n], n>0$ act by zero. This in particular implies the existence of a non vanishing maximal vector $v_{+} \in V_{0}$ such that

$$
\begin{aligned}
e[0] v_{+} & =0, \\
h[0] v_{+} & =\lambda v_{+}, \\
\kappa v_{+} & =c v_{+},
\end{aligned}
$$

where $\lambda \in \mathbb{Z}_{+}, c \in \mathbb{C}$. Construct the vector space

$$
W=U(\widehat{s l}(2)) \otimes_{U(\mathfrak{p})} V_{0}
$$

and define a left $\widehat{s l}(2)$ action on it by the multiplication of $U(\widehat{s l}(2))$, thus turning $W$ into a $U(\widehat{s l}(2))$ module, which will be called a generalized Verma module. It is clearly true that $W$ is $U\left(\mathfrak{u}_{+}\right)$- locally finite, i.e., for any $w \in W$ and $u \in \mathfrak{u}_{+}$, there exists a finite positive integer $N$ such that $(u)^{N} w=0$. Also, it is a direct consequence of the Poincaré - Birkhoff - Witt theorem that $W=U\left(\mathfrak{u}_{-}\right) \otimes_{\mathbb{C}} V_{0}$ as a vector space. In general, $W$ itself is not irreducible, but contains a unique maximal proper submodule $M$ such that the quotient

$$
V=W / M
$$

gives rise to an irreducible $U(\widehat{s l}(2))$ module. When $c-\lambda \in \mathbb{Z}_{+}, V$ is integrable, and its structure is well understood [6].

From here on we will assume that $c \in \mathbb{R}$. This includes the integrable highest weight modules as special cases. Now we can give a relatively easy and explicit characterization of the maximal proper submodule $M$. Recall that $\widehat{s l}(2)$ admits the following anti - linear involution( denoted by $\dagger$ )

$$
\begin{aligned}
(\kappa)^{\dagger} & =\kappa, \\
(e[n])^{\dagger} & =f[-n], \\
(f[n])^{\dagger} & =e[-n], \\
(h[n])^{\dagger} & =h[-n], \quad n \in \mathbb{Z} .
\end{aligned}
$$

Using it, we can define a sesquilinear form $\langle\mid\rangle: W \times W \rightarrow \mathbb{C}$ by requiring

i). $\left.\langle\mid\rangle\right|_{V_{0} \otimes V_{0}}$ defines a Hermitian inner product for $V_{0}$ regarded as a module of $\operatorname{sl}(2)=\langle e[0], h[0], f[0]\rangle$, and $\left\langle v_{+} \mid v_{+}\right\rangle=1$;

ii). $\left\langle u w_{1} \mid w_{2}\right\rangle=\left\langle w_{1} \mid(u)^{\dagger} w_{2}\right\rangle, \quad \forall w_{1}, w_{2} \in W, u \in U(\widehat{s l}(2))$. 
It is a straightforward exercise to show that $\langle\mid\rangle$ is well defined and unique. Furthermore,

$$
\begin{aligned}
M & =\operatorname{Ker}\langle\mid\rangle \\
& :=\{v \in W \mid\langle v \mid W\rangle=0\} .
\end{aligned}
$$

\section{VECTOR COHERENT STATES}

We now turn to the vector coherent state method, which, when restricted to integrable highest weight irreps, is the infinitesimal version of the construction of Pressley and Segal's [10], which yields integrable highest weight irreps of loop groups as holomorphic sections of vector bundles over 'fundamental homogeneous spaces'. Introduce the indeterminates $x_{k}, y_{k}, z_{k}, k=1,2, \ldots, \infty$, and denote by $P(X, Y, Z)$ the linear span of their polynomials with coefficients in $\mathbb{C}$. Denote

$$
\begin{aligned}
& E(X)=\sum_{k=1}^{\infty} e[k] x_{k}, \\
& H(Y)=\sum_{k=1}^{\infty} h[k] y_{k}, \\
& F(Z)=\sum_{k=1}^{\infty} f[k] z_{k} .
\end{aligned}
$$

Introduce the formal power series, $\exp (E(X)), \exp (H(Y))), \exp (F(Z))$, of the indeterminates with coefficients in $U(\widehat{s l}(2))$, where, e.g.,

$$
\exp (E(X))=\sum_{n=0}^{\infty}(E(X))^{n} / n !
$$

Let

$$
g(X, Y, Z)=\exp (E(X)) \exp (H(Y))) \exp (F(Z))
$$

Following the strategy of the vector coherent state method for ordinary Lie algebras [1, 2, 3, 5], we define the linear map $\xi(X, Y, Z): W \rightarrow P(X, Y, Z) \otimes_{\mathbb{C}} V_{0}$ by

$$
\xi_{w}(X, Y, Z)=\sum_{i=1}^{\operatorname{dim} V_{0}}\left\langle v_{i}|g(X, Y, Z)| w\right\rangle \otimes v_{i}, \quad w \in W
$$

where $\left\{v_{i} \mid i=1,2, \ldots, \operatorname{dim} V_{0}\right\}$ forms a basis of $V_{0}$ such that $\left\langle v_{i} \mid v_{j}\right\rangle=\delta_{i j}$, the existence of which is guaranteed by the finite dimensionality of $V_{0}$ as an $s l(2)$ module. We denote by $\boldsymbol{\Xi}(X, Y, Z)$ the image of the map, and call it the space of the vector coherent states. Note that $\Xi(X, Y, Z)$ is indeed a subset of $P(X, Y, Z) \otimes_{\mathbb{C}} V_{0}$ because of the $U\left(\mathfrak{u}_{+}\right)$local finiteness of $W$.

It is of crucial importance to observe that the coefficients of the formal power series $g(X, Y, Z)$ in the variables $x_{k}, y_{k}, z_{k}, k=1,2, \ldots, \infty$, form a basis of $U\left(\mathfrak{u}_{+}\right)$. Therefore, an element $w \in W$ belongs to the maximal proper submodule $M \subset W$ if and only if $\xi_{w}(X, Y, Z)=0$. Also, $\Xi(X, Y, Z)$ admits a natural $U(\widehat{s l}(2))$ action

$$
u \cdot \xi_{w}(X, Y, Z)=\xi_{u w}(X, Y, Z), \quad u \in U(\widehat{s l}(2)), w \in W
$$


Now we have the following results:

$$
\begin{array}{ll}
\text { i). } & \operatorname{Ker} \xi(X, Y, Z)=M \\
i i) . & \Xi(X, Y, Z) \cong V \text { as irreducible } U(\widehat{s l}(2)) \text { modules. }
\end{array}
$$

One of the advantages of the vector coherent state formulation of representations is that the elements of the affine Lie algebra can be realized explicit in terms of differential operators on $P(X, Y, Z)$ and endomorphisms of $V_{0}$. To obtain such a realization, we need some technical results, which we now discuss. Define the shifting operators $\sigma_{n}$, $n \in \mathbb{Z}$ by

$$
\begin{aligned}
\sigma_{n} E(X) & =\sum_{k=1}^{\infty} e[n+k] x_{k}, \\
\sigma_{n} H(Y) & =\sum_{k=1}^{\infty} h[n+k] y_{k}, \\
\sigma_{n} F(Z) & =\sum_{k=1}^{\infty} f[n+k] z_{k},
\end{aligned}
$$

and also introduce the polynomials

$$
\mathbf{Z}_{N}(Y)=\left.\frac{1}{N !} \frac{d^{N}}{d t^{N}} \exp \left(\sum_{k=1}^{\infty} t^{k} y_{k}\right)\right|_{t=0}, \quad N \in \mathbb{Z}_{+} .
$$

Some lengthy, but relatively straightforward, calculations yield the following relations,

$$
\begin{aligned}
{[\exp (E(X)), h[k]] } & =-2 \exp (E(X)) \sigma_{k} E(X), \\
{[\exp (H(Y))), h[k]] } & \left.=-2 \kappa k \theta(-k) y_{-k} \exp (H(Y))\right), \\
{[\exp (F(Z)), h[k]] } & =2 \exp (F(Z)) \sigma_{k} F(Z), \\
f[k] \exp (H(Y))) & =\exp (H(Y))) \sum_{N=0}^{\infty} \mathbf{Z}_{N}(2 Y) f[k+N], \\
{[\exp (E(X)), f[k]] } & =\exp (E(X))\left\{\sigma_{k} H(X)-k \kappa x_{-k} \theta(-k)+\sum_{p=1}^{\infty} x_{p} \sigma_{p+k} E(X)\right\}, \\
{[\exp (F(Z)), e[k]] } & =\exp (F(Z))\left\{\sum_{p=1}^{\infty} z_{p} \sigma_{p+k} F(Z)-\kappa k z_{-k} \theta(-k)-\sigma_{k} H(Z)\right\}, \\
\exp (H(Y)) e[k] & =\sum_{N=0}^{\infty} \mathbf{Z}_{N}(2 Y) e[k+N] \exp (H(Y)), \quad k \in \mathbb{Z},
\end{aligned}
$$

where $2 Y=\left(2 y_{1}, 2 y_{2}, \ldots\right)$, and $\theta(k)= \begin{cases}1, & k>0 \\ 0, & k \leq 0\end{cases}$

With the help of these relations, we can readily obtain the realization of the elements of the affine Lie algebra $\widehat{s l}(2)$ in explicit form. We will denote the realization by $\xi$. For the simple and Cartan generators, we have

$$
\xi(\kappa)=c
$$




$$
\begin{aligned}
\xi(f[1]) & =\frac{\partial}{\partial z_{1}}, \\
\xi(h[0]) & =\pi_{0}(h)+2 \sum_{p=1}^{\infty}\left[z_{p} \frac{\partial}{\partial z_{p}}-x_{p} \frac{\partial}{\partial x_{p}}\right] \\
\xi(e[0]) & =\pi_{0}(e)+\sum_{N=1}^{\infty} \mathbf{Z}_{N}(2 Y) \frac{\partial}{\partial x_{N}}-\sum_{p=1}^{\infty}\left[z_{p} \frac{\partial}{\partial y_{p}}+z_{p} \sum_{q=1}^{\infty} z_{q} \frac{\partial}{\partial z_{p+q}}\right] \\
\xi(f[0]) & =\pi_{0}(f)-\sum_{N=1}^{\infty} \mathbf{Z}_{N}(2 Y) \frac{\partial}{\partial z_{N}}+\sum_{p=1}^{\infty}\left[x_{p} \frac{\partial}{\partial y_{p}}+x_{p} \sum_{q=1}^{\infty} x_{q} \frac{\partial}{\partial x_{p+q}}\right] \\
\xi(e[-1]) & =2 y_{1} \pi_{0}(e)+z_{1}\left\{c-\pi_{0}(h)+2 \sum_{p=1}^{\infty} x_{p} \frac{\partial}{\partial x_{p}}\right\} \\
& +\sum_{N=1}^{\infty} \mathbf{Z}_{N+1}(2 Y) \frac{\partial}{\partial x_{N}}-\sum_{p=1}^{\infty}\left\{z_{p+1} \frac{\partial}{\partial y_{p}}+z_{p} \sum_{q=1}^{\infty} z_{q} \frac{\partial}{\partial z_{p+q-1}}\right\}
\end{aligned}
$$

where $\pi_{0}(e), \pi_{0}(h), \pi_{0}(f) \in \operatorname{End}\left(V_{0}\right)$ are respectively defined by the actions of $e[0], h[0]$ and $f[0]$ on $V_{0}$.

In principle, these elements are sufficient to generate the entire $\widehat{s l}(2)$ algebra. However, it is not much more difficult to work out the realization for other generators, which we spell out below:

$$
\begin{aligned}
\xi(f[k]) & =\frac{\partial}{\partial z_{k}}, \\
\xi(h[k]) & =\frac{\partial}{\partial y_{k}}+2 \sum_{p=1}^{\infty} z_{p} \frac{\partial}{\partial z_{k+p}}, \\
\xi(e[k]) & =\frac{\partial}{\partial x_{k}}+\sum_{N=1}^{\infty} \mathbf{Z}_{N}(2 Y) \frac{\partial}{\partial x_{k+N}}-\sum_{p=1}^{\infty}\left[z_{p} \frac{\partial}{\partial y_{k+p}}+z_{p} \sum_{q=1}^{\infty} z_{q} \frac{\partial}{\partial z_{k+p+q}}\right], \\
\xi(f[-k]) & =\mathbf{Z}_{k}(-2 Y) \xi(f[0])+\sum_{t=1}^{k} \mathbf{Z}_{k-t}(-2 Y) \mathcal{D}_{t}, \\
\xi(h[-k]) & =2 c k y_{k}-2 x_{k} \pi_{0}(e)+2 \sum_{p=1}^{\infty}\left[z_{k+p} \frac{\partial}{\partial z_{p}}-x_{k+p} \frac{\partial}{\partial x_{p}}\right]+2 x_{k} \sum_{p=1}^{k} z_{p} \xi(f[p-k]), \\
\xi(e[-k]) & =c k z_{k}+\mathbf{Z}_{k}(2 Y) \pi_{0}(e)+\sum_{N=1}^{\infty} \mathbf{Z}_{N+k}(2 Y) \frac{\partial}{\partial x_{N}} \\
& -\xi\left(\sigma_{-k} H(Z)\right)+\sum_{p=1}^{\infty} z_{p} \xi\left(\sigma_{p-k} F(Z)\right),
\end{aligned}
$$

where

$$
\begin{aligned}
\mathcal{D}_{k} & =x_{k}\left(c k+\pi_{0}(h)\right) \\
& -\sum_{N=1}^{\infty} \mathbf{Z}_{N+k}(2 Y) \frac{\partial}{\partial z_{N}}+\sum_{p=1}^{\infty} x_{p+k}\left(\frac{\partial}{\partial y_{p}}+\sum_{q=1}^{\infty} x_{q} \frac{\partial}{\partial x_{p+q}}\right) \\
& -\sum_{p=1}^{\infty} \sum_{q=1}^{k} x_{p} x_{q}\left\{\theta(p+q-k) \frac{\partial}{\partial x_{p+q-k}}+\delta_{p+q, k} \pi_{0}(e)\right\}, \quad k>0 .
\end{aligned}
$$


We can also introduce the grading operator $d$. It necessarily acts on $V_{0}$ by a constant $d_{0} \in \mathbb{R}$, which can in fact be chosen arbitrarily. Now

$$
\xi(d)=d_{0}-\sum_{p=1}^{\infty} p\left(x_{p} \frac{\partial}{\partial x_{p}}+y_{p} \frac{\partial}{\partial y_{p}}+z_{p} \frac{\partial}{\partial z_{p}}\right) .
$$

An important feature of the vector coherent state realization is that repeated applications of $\xi(e[-k]), \xi(h[-k]), \xi(f[-k]), k>0$, to $1 \otimes V_{0}$ will automatically generate the irreducible $U(\widehat{s l}(2))$ module $\boldsymbol{\Xi}(X, Y, Z)$. Also observe that $\xi(\widehat{s l}(2))$ acts naturally on $\bar{W}(X, Y, Z)=P(X, Y, Z) \otimes V_{0}$, and $\Xi(X, Y, Z)$ is contained in $\bar{W}(X, Y, Z)$ as the unique minimal submodule. It appears possible to extend the $\mathrm{K}$ - matrix technique[2] to the present context to explicitly identify this submodule. A different approach is to find, by cohomological means, a system of linear differential operators with constant coefficients acting on $\bar{W}(X, Y, Z)$, the kernel of which will reproduce $\boldsymbol{\Xi}(X, Y, Z)$. We will report our results on these problems in a separate publication, where the coherent state method is also developed for general affine Lie algebras.

\section{References}

[1] K. T. Hecht, The vector coherent state method and its applications to problems in higher symmetries, Lecture Notes in Physics, 290 Springer - Verlag, Berlin(1987), and references therein.

[2] D. J. Rowe and J. Repka, J. Math. Phys. 32 (1991) 2614.

[3] R. Le Blanc and D. J. Rowe, J. Math. Phys. 30 (1989) 1415;

C. Quesne, J. Phys. A 23 (1990) 5383.

[4] L. C. Biedenharn and M. Lohe, Comm. Math. Phys. 146 (1992) 483;

R. B. Zhang, Bott - Borel - Weil construction for quantum supergroup $U_{q}(g l(m \mid n))$, J. Math. Phys., in press.

[5] D. J. Rowe, G. Rosensteel and R. Gilmore, J. Math. Phys. 26 (1985) 2787.

[6] V. G. Kac, Infinite dimensional Lie algebras, 3rd edition, Cambridge press, Melbourne (1990).

[7] P. Goddard and D. Olive, Kac - Moody and Virasoro algebras, World Scientific, Singapore (1988).

[8] C. Itzykson, H. Saleur and J. - B. Zuber, Conformal invariance and applications to statistical mechanics, World Scientific, Singapore (1988).

[9] E. Fradkin, Field theories of condensed matter systems, Addison - Wesley Publishing Company (1991).

[10] A. Pressley and G. Segal, Loop groups, Clarendon Press, Oxford (1986).

[11] B. Feigin and E. Frenkel, Comm. Math. Phys. 128 (1990) 161. 